Recepción: 11 / 04 / 2018

Aceptación: 26 / 05 / 2018

Publicación: 02 / 07 / 2018

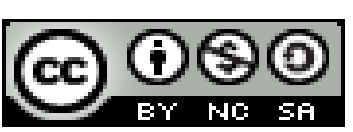

Ciencias sociales y políticas

Artículo de Investigación

\title{
Liderazgo y educación universitaria
}

\section{Leadership and university education}

\section{Liderança e educação universitária}

José V. Lucas-Muentes I jose.lucas@hotmail.com

Monis R. Mendoza-Andrade II monis.mendoza@hotmail.com

Arsenio H. Lucas-Muentes III arse.lucas@hotmail.com

Correspondencia: arse.lucas@ hotmail.com

\footnotetext{
I Master en Ciencias Especialidad Docencia Universitaria, Doctor en Ciencias de la Educación Mención Enseñanza de la Matemática, Licenciado en Ciencias de la Educación Profesor de Enseñanza Secundaria en la Especialización de Físico Matemáticas, Docente de la Universidad Técnica Luis Vargas Torres de Esmeraldas, Esmeraldas, Ecuador.

II Magister en Desarrollo Humano y Comunitario, Licenciado en Trabajo Social, Docente de la Universidad Técnica Luis Vargas Torres de Esmeraldas, Esmeraldas, Ecuador.

III Master en Ciencias Especialidad Docencia Universitaria, Licenciado en Ciencias de la Educación; Profesor de Enseñanza Secundaria en la Especialización de Físico Matemáticos, Doctor en Ciencias De La Educación Mención Enseñanza de la Matemática, Docente de la Universidad Técnica Luis Vargas Torres de Esmeraldas, Esmeraldas, Ecuador.
} 


\title{
Resumen
}

El presente artículo resulta en una revisión signada por la sociología política, del fenómeno del liderazgo en la educación universitaria. Se parte de una revisión bibliográfica corta acerca de diferentes enfoques teóricos acerca del liderazgo, reconociendo que tal categoría tiene múltiples acepciones, caracterizaciones y tipologías, empezando por las teorías de los rasgos, predominante en las primeras épocas del pensamiento académico. Se revisan algunas explicaciones desde la psicología y la sociología, privilegiando esta última de las miradas desde el enfoque weberiano enriquecido por French y Raven (1968). Seguidamente se examina la organización universitaria, con énfasis en el marco organizacional y simbólico, para determinar su carácter complejo y de "anarquía organizacional", para finalmente explorar diversas formas de concreción del establecimiento del liderazgo en las universidades, mediante la diversidad de métodos y actores involucrados en la selección del rector y como se traduce en diferenciada fuente de legitimidad y poder en tal liderazgo. Se concluye estableciendo que el liderazgo en la educación universitaria responde en su caracterización a la pluralidad de modelos complejos que existen en las organizaciones universitarias, tendiendo a ser predominantes dos modelos: el meritocrático o de poder experto y el carismático.

Palabras clave: liderazgo; gestión; educación universitaria; organización universitaria; legitimidad.

\begin{abstract}
The present article results in a revision marked by political sociology, the phenomenon of leadership in university education. It is based on a short bibliographic review about different theoretical approaches to leadership, recognizing that this category has multiple meanings, characterizations and typologies, starting with the theories of the traits, predominant in the early eras of academic thought. Some explanations are reviewed from psychology and sociology, privileging the latter from the views from the Weberian approach enriched by French and Raven (1968). Next, the university organization is examined, with emphasis on the organizational and symbolic framework, to determine its complex character and "organizational anarchy", to finally explore different ways to concretize the establishment of leadership in universities, through the diversity of methods and actors involved in the selection of the rector and how it translates into a differentiated source of legitimacy and power in such leadership. It concludes establishing that
\end{abstract}


leadership in university education responds in its characterization to the plurality of complex models that exist in university organizations, tending to be predominantly two models: the meritocratic or expert power and the charismatic.

Keywords: Challenges; higher education; Latin America; XXI century.

\section{Resumo}

O presente artigo resulta em uma revisão marcada pela sociologia política, fenômeno da liderança no ensino universitário. É parte de uma curta revisão da literatura sobre as diferentes abordagens teóricas sobre liderança, reconhecendo que tal categoria tem múltiplos significados, caracterização e tipologias, começando com as teorias de traços, predominantes nos primórdios do pensamento acadêmico. Algumas explicações são revisadas a partir da psicologia e sociologia, privilegiando as últimas a partir das visões da abordagem weberiana enriquecida por French e Raven (1968). Em seguida, a organização universitária é examinado, com ênfase na estrutura organizacional e simbólico para determinar seu caráter complexo e "organizacional anarquia", para finalmente explorar várias formas de realização do estabelecimento de liderança nas universidades, através da diversidade de métodos e atores envolvido na seleção do reitor e como se traduz em uma fonte diferenciada de legitimidade e poder em tal liderança. Conclui-se que a liderança no ensino superior responde à caracterização pluralidade de modelos complexos que existem em organizações universitárias, que tende a predominar dois modelos: o poder meritocrática ou perito e carismático.

Palavras chave: liderança; gestão; educação universitária; organização universitária; legitimidade.

\section{Introducción}

La educación universitaria tiene su asiento en las organizaciones conocidas como Universidades. Estas organizaciones son dirigidas por líderes cuyo poder o fuente de legitimidad tiende a ser diverso en la medida en que existe diversidad en el modo en que son designados los rectores.

El liderazgo es una categoría que ha sido estudiada por diferentes disciplinas, encontrándose múltiples definiciones, de las cuales la más antigua y que aún mantiene vigencia es la que refiere 
a las cualidades especiales e innatas de los líderes, es decir la teoría del gran hombre o héroe, y la de los rasgos.

El enfoque de la sociología política, que destaca en el liderazgo los aspectos que tienen que ver con el poder, la dominación, la legitimidad, están muy influidos por la teoría de los modelos de dominación de Max Weber, la cual ha sido enriquecida por otros autores.

Se revisa los aportes de Ordonika $(1995,2001)$ en sus estudios acerca de la Universidad como organización, su complejidad, para reflexionar acerca de las modalidades de liderazgo que se desprenden tanto de la pluralidad de la naturaleza de sus funciones (docencia, investigación, extensión, gerencia universitaria y gremial) como de la diversidad de formas de designación de las altas autoridades en las diferentes universidades del mundo, debatiéndose el predominio de dos tipos de liderazgo, fundamentalmente: el meritocrático o de poder experto y el carismático.

\section{Enfoques acerca del liderazgo}

El tema del liderazgo es ya un tema clásico. Su estudio ha sido abordado en múltiples disciplinas, así como de manera interdisciplinaria y transdisciplinaria. Presentándose a lo largo de la historia una gama considerable de interpretaciones, clasificaciones y tipificaciones del fenómeno, habiendo sido distinguido enfoques que privilegian su estudio desde la conducta, los rasgos de la personalidad, el poder (influencia, dominación, autoridad), arte, cambio, gerencia, entre otros.

La mayor parte de las reseñas (Labourdette \& Scaricabarozzi: 2010 entre otros) ubican como primeras clasificaciones del fenómeno en estudio aquellas que se afincan en las cualidades o rasgos personales del actor que ejerce el liderazgo. Pueden rastrearse estudios (Silva, 2016) que indican que ya para unos 2.500 años A.C. el sabio de origen chino Confucio planteaba algo similar, como Platón en Grecia antigua y ya en los inicios del pensamiento moderno Maquiavelo, estableciendo las necesarias virtudes que debía presentar quien ejerciera el liderazgo.

En Psicología, ubican los estudios predominantemente orientados así en las décadas de los años 30 y 40 del siglo XX, aun cuando también afirma Silva (2016) que en el siglo XIX fue popularizado el enfoque que fue denominado el del gran hombre, considerando a los líderes como héroes que lograban alcanzar poder e influencia en otros hombres. 
En sociología, específicamente desde una perspectiva micro sociológica, no puede dejarse de mencionar la teoría de los modelos de dominación de Weber (2002), la cual conecta directamente la noción de liderazgo a la de dominación, de legitimidad, y a su vez al tipo de acción social predominante, desde su contínuum de la racionalidad (finalista y valorativa) a la no racionalidad (afectiva y tradicional). Es así como deriva sus tres tipos de liderazgo: carismático (la fuente de legitimidad está en la capacidad del líder de mover las emociones), tradicional (la fuente de legitimidad está en la creencia en el origen divino) y racional legal (con fuente de legitimidad fundamentado en la norma). (Weber, 2002, p. 173)

De manera tal que el liderazgo es concebido desde la perspectiva weberiana, como una relación social de poder o dominación entre un actor individual respecto a otro actor individual o colectivo, ejerciendo la dominación legítima mediante acciones sociales sucesivas en las que se combinan las diversas fuentes de legitimidad de la intencionalidad del primero, generando la probabilidad de obediencia a una orden o un orden por parte de los segundos. Específicamente en la sociedad moderna, contemporánea y actual se identifica el predominio de la forma racional legal de fuente de legitimidad, aun cuando necesariamente se combina en diversos grados manifestaciones de las otras formas de dominación. (Weber, 2002)

En Psicología se ha discutido con mucha vehemencia diferentes enfoques que se debaten entre las cualidades personales del líder, las influencias de situaciones contextuales, el modo como afronta el líder las cosas o las cosas que hace, siempre en la búsqueda de valorar quien ejercería el mejor liderazgo. Así, Shultz (1995) identifica al menos dos enfoques contrapuestos que han dividido las opiniones de los investigadores en la ciencia psicológica, el enfoque de rasgos y el enfoque situacional. El primero se sustenta en el supuesto de que los líderes son personas que nacen con cualidades específicas que los predisponen a ejercer liderazgo efectivo, aun cuando afirma el autor de que no ha podido ser demostrado fehacientemente cuales son las características comunes en los líderes efectivos. Mientras que el situacional supone que es posible propiciar el desarrollo de habilidades para el ejercicio del liderazgo eficaz. Para luego concluir luego de desarrollar la contradicción entre ambos enfoques, que

En conclusión, sus conductas y cualidades, las características de los seguidores y la naturaleza de la situación en que interactúan unos y otros son aspectos individuales, pero independientes en el 
proceso del liderazgo. Hay que analizarlos todos si queremos entender y estudiar su naturaleza y exigencia. (Shultz, 1995, p. 218)

De hecho, la postura de Shultz (1995 expresa lo que según Silva (2016) ocurrió en el mundo de la investigación psicológica referida a este fenómeno, cuando se fue migrando hacia la mirada del liderazgo más como una relación o interacción en la cual los seguidores o colaboradores juegan también un papel relevante en la eficacia del liderazgo como fenómeno de carácter social.

Ahora bien, luego del esfuerzo analítico y sintético realizado por Silva (2016) a la noción de liderazgo (desde una perspectiva psicológica) este autor concluye que se ha demostrado que el liderazgo es un concepto en evolución, pero podría ser satisfactoriamente definido como "el proceso de influencia interactiva que ocurre cuando, en un contexto dado, algunas personas aceptar a alguien como su líder para alcanzar objetivos comunes " (Silva, 2016). Esta definición parece ajustarse adecuadamente al concepto moderno de liderazgo, que le da líder, los seguidores y el contexto un papel muy importante en el proceso de liderazgo.

Mientras tanto la perspectiva sociológica también evolucionó a partir de la idea expuesta anteriormente por Weber (2002), enfocándose a partir de la perspectiva interaccionista en el tema central del poder en el proceso del vínculo social (Marín, 1997)

Así, Monzón (en Marín, 1997) cita a French y Raven (1968), quienes exponen una ampliación a la tipificación del liderazgo en tanto dominación que había propuesto Weber con sus tres tipos puros, llevándolos a cinco de la siguiente manera:

Tabla 1. Liderazgo a partir del Poder

\begin{tabular}{|c|c|c|}
\hline Tipo de poder & Origen & Bases \\
\hline Premio o recompensa & $\begin{array}{l}\text { Ofrecimiento de algo valioso a } \\
\text { cambio de sumisión }\end{array}$ & El beneficio \\
\hline Experto & $\begin{array}{l}\text { Experiencia, conocimiento o } \\
\text { habilidades especiales del líder }\end{array}$ & Información \\
\hline Referente o carismático & Cualidades y atractivos del líder & $\begin{array}{l}\text { Capacidad del líder de mover } \\
\text { emociones }\end{array}$ \\
\hline Coercitivo & Amenaza & Miedo \\
\hline
\end{tabular}




\begin{tabular}{|l|l|l|}
\hline Legítimo & Criterios de legitimación & $\begin{array}{l}\text { Razones de investidura } \\
\text { aceptada voluntariamente por } \\
\text { el seguidor }\end{array}$ \\
\hline
\end{tabular}

Fuente: Monzón en Marín (1997, p. 50)

A pesar de que no se trata de las únicas propuestas de explicación y tipificación del liderazgo como categoría, se considera que para los efectos del presente artículo es un referente bastante adecuado, por lo que se asume como su fundamentación más inmediata.

\section{La educación universitaria y los liderazgos}

El ámbito organizacional de la educación universitaria es la Universidad. Vista como institución sería el modo y ámbito legítimo para el acceso al conocimiento elevado y profundo en lo técnico, científico, artístico y humanístico.

Como organización ha sido considerada como uno de los tipos más conservadores, complejos y resistentes al cambio (Ordorika, 1995). Citando a Dahrendorf, 1979), este autor reseña la enorme autonomía de la que disfruta la organización universitaria en el ámbito de las sociedades actuales. Resulta ser una combinación de tensiones entre actores organizacionales de diferente naturaleza: los docentes (dedicados a la actividad académica propiamente tal, incluyendo aquellos que ejercen el gobierno y co gobierno universitario), el personal administrativo (la burocracia universitaria), los estudiantes y el personal obrero de mantenimiento.

Algunos teóricos caracterizan a las organizaciones académicas como instituciones "procesadoras de personas". Individuos con necesidades específicas se incorporan a la organización partiendo de su medio ambiente, la organización actúa sobre ellos y estos son regresados a la sociedad. Es una condición muy especial de estas organizaciones. Los beneficiarios son parte de la organización y participan en la toma de decisiones dentro de ella. (Ordorika 1995, p. 5)

En este aspecto, se privilegia en la apreciación acerca de la universidad como organización su papel como formadora de profesionales, técnicos, científicos y humanistas para el desarrollo del resto de las organizaciones públicas y privadas de la sociedad. Es decir, una organización al servicio de la sociedad hacia afuera y al servicio del sector estudiantil hacia adentro. 
Pero son también un pesado aparato administrativo sometido a la típica lógica burocrática: extrema y complicada división de competencias a manera de división del trabajo, jerarquización de las funciones y los roles, carrera administrativa en aquellas oficinas dedicadas al manejo presupuestario, administrativo, del talento humano. "Pero no tienen otros atributos, por ejemplo, no hay supervisión directa del trabajo de la mayoría de los empleados (los profesores), y no hay reglas operativas detalladas que rijan el desempeño de las responsabilidades académicas" (Blau, 1973, citado por Orinka , 2001, p. 86).

Visto desde una perspectiva de marco simbólico (Pfeffer 1981, Bensimon 1989), es decir, en tanto sistemas culturales, que involucran significados y creencias, nos refiere Ordorika (1995) que Cohen y March caracterizan a las universidades como "anarquías organizadas" como consecuencia de sus metas problemáticas, su tecnología poco clara, y la participación fluida de sus miembros:

En una anarquía universitaria cada individuo en la universidad es visto como tomando decisiones autónomas. Los maestros deciden sí enseñan, cuándo enseñan y qué enseñan. Los estudiantes deciden sí aprenden, cuándo aprenden y qué aprenden. Los consejeros universitarios y los patrones deciden sí apoyan, cuándo apoyan y qué apoyan. (Ordorika, 1995 citando a Cohen y March 1974, p. 8)

La complejidad de esta organización deriva quizás de la variedad en la naturaleza de sus funciones básicas: Docencia, Investigación, Extensión, Gerencia Universitaria. Esta última pretende vincular y coordinar las diferentes funciones, y operaciones organizacionales, manteniéndose en tensión con el resto de las funciones. Esto en combinación con la diversidad de actores colectivos (estudiantes, obreros, docentes, personal administrativo). Tales colectivos generalmente organizados en grupos de presión (gremios, sindicatos), con adición a sus representaciones en los organismos colegiados de cogobierno universitario, en los cuales se toman las decisiones que están dentro de los límites de la autonomía normativa y administrativa de las Universidades. Todo ello da pie a diversidad de expresiones de liderazgo que se conjugan en el juego de interacciones de la vida universitaria.

A partir de esto último, se perfila el sistema político a lo interno de la Universidad como espacio organizacional e institucional de la educación universitaria. La Universidad asume la forma de 
una república sui géneris en la que la selección de quienes ejercerán gran parte de los espacios de liderazgo es sometida a procesos eleccionarios, teniendo como premisa la democracia representativa (Arias, 2012, p.45). Lo especial está en que los “ciudadanos" sometidos al gobierno universitario conforman una complicada comunidad marcada por una desigualdad estamentarizada: en muchos casos los únicos que tienen derecho por ley a ejercer como autoridades universitarias son los profesores miembros del claustro universitario, compartiendo sólo con los estudiantes el privilegio de ser electores bajo una desigualdad marcada, en algunos casos desde 50 o $60 \%$ del peso para los docentes, mientras que la diferencia la comparten los estudiantes (cerca de un $30 \%$ ) y los egresados (10\%), en otros casos de aproximadamente 25 votos estudiantiles por cada voto docente.

Al llevar a cabo una rápida revisión de los métodos de designación de rector en otras latitudes y, en especial, en universidades de alto reconocimiento académico, nos encontramos con que, en contra de lo que pueda pensarse, son más numerosas las universidades que, en uso de su autonomía, optan por métodos de designación de sus directivas concentrando la responsabilidad de la decisión en unos pocos integrantes de la comunidad académica que conforman estamentos colegiados de amplia respetabilidad y profundo conocimiento de la universidad, de su entorno y de sus proyecciones. (Mantilla, 2018)

Según este autor, la tendencia mayoritaria en las universidades del mundo es la escogencia del líder de la Universidad, es decir al Rector, privilegiando la pesquisa de entre los más calificados académicamente, tratando de evitar que se cuelen liderazgos carismáticos que sean electos más bien por su popularidad.

Tabla 2. Formas de elegir autoridades rectorales

\begin{tabular}{|l|l|l|l|}
\hline País & Universidad & $\begin{array}{l}\text { Forma de elección del } \\
\text { Rector }\end{array}$ & Quienes eligen \\
\hline Colombia & $\begin{array}{l}\text { Universidad Nacional de } \\
\text { Colombia }\end{array}$ & $\begin{array}{l}\text { Consulta electrónica } \\
\text { ponderada:60\% peso de los } \\
\text { profesores, 30\% peso de los } \\
\text { estudiantes, Egresados 10\%. } \\
\text { El Consejo Superior elige } \\
\text { entre los 5 más respaldados }\end{array}$ & $\begin{array}{l}\text { Consejo Superior de } \\
\text { la Universidad }\end{array}$ \\
\hline México & $\begin{array}{l}\text { Universidad Nacional } \\
\text { Autónoma de México }\end{array}$ & $\begin{array}{l}\text { Consulta a entes } \\
\text { universitarios, entrevistas }\end{array}$ & $\begin{array}{l}\text { La Junta de Gobierno } \\
\text { de la Universidad }\end{array}$ \\
\hline
\end{tabular}




\begin{tabular}{|l|l|l|l|}
\hline & & $\begin{array}{l}\text { con candidatos y } \\
\text { personalidades académicas }\end{array}$ & \\
\hline EEUU & Universidad de Harvard & $\begin{array}{l}\text { Comité de búsqueda propone } \\
\text { candidatos (método } \\
\text { compartido por la mayoría } \\
\text { de las universidades } \\
\text { norteamericanas y europeas) }\end{array}$ & La corporación \\
\hline Brasil & $\begin{array}{l}\text { Universidad de Sao } \\
\text { Paulo }\end{array}$ & $\begin{array}{l}\text { Votación ponderada con } \\
\text { fuerte predominio de } \\
\text { profesores, proponen terna }\end{array}$ & $\begin{array}{l}\text { El gobernador del } \\
\text { estado de Sao Paulo } \\
\text { designa al Rector }\end{array}$ \\
\hline Chile & Universidad de Chile & $\begin{array}{l}\text { votación de los profesores de } \\
\text { las tres más altas jerarquías y } \\
\text { mayor antigüedad proponen } \\
\text { terna }\end{array}$ & $\begin{array}{l}\text { Designa el presidente } \\
\text { de la Republica }\end{array}$ \\
\hline España & $\begin{array}{l}\text { Universidad de } \\
\text { Salamanca }\end{array}$ & $\begin{array}{l}\text { Elección por parte de } \\
\text { Profesores (peso superior a } \\
50 \%) \text { y estudiantes (30\%) }\end{array}$ & $\begin{array}{l}\text { Docentes y } \\
\text { estudiantes }\end{array}$ \\
\hline
\end{tabular}

Fuente: Mantilla (2018)

\section{El liderazgo en la educación universitaria}

De manera pues que la caracterización del liderazgo en la educación universitaria responde a la complejidad que caracteriza a estas organizaciones, presentándose más bien una serie de combinaciones de sus fuentes de legitimidad y origen. Es así que entonces, en las organizaciones universitarias tocadas por la conquista de democratización a partir de la Reforma Universitaria de Córdova, la fuente y origen de la legitimidad responderá en la forma a la racionalidad formal legal que otorgue la ley, pero en el fondo, dada la modalidad eleccionaria típica de la democracia representativa, tenderán a predominar aquellos liderazgos que alcancen mayor popularidad (poder referente) tanto entre los docentes (que tienen el mayor peso en la elección) y en los estudiantes (cuyo peso tiende a estar en el orden del $30 \%$ ), por tanto el sesgo político partidario tiende a imponerse por encima de las consideraciones meritocráticas (poder experto). Tal situación aplica también para los decanatos y espacios de cogobierno a los cuales elegirán los estudiantes, profesores y egresados sus respectivos representantes, así como para los espacios de orden gremial y sindical y centros y federaciones de centros universitarios. Sin embargo, de acuerdo a las fuentes consultadas no es esta la situación mayoritaria en las universidades norteamericanas y europeas en las cuales, siendo más conservadoras y por tanto menos democráticas, serían órganos superiores de gobierno universitario los que a partir de los principios de la meritocracia designarían a las máximas autoridades universitarias. 
Otros espacios organizacionales de la Universidad como las Cátedras y Departamentos tenderán a responder más al criterio de méritos académicos alcanzados por los docentes que se postulen, a semejanza de ocurrido en los espacios de la investigación y la extensión universitaria. Por su parte, los liderazgos de los espacios propiamente de la gestión administrativa serán designados por los órganos superiores universitarios, combinando diferentes fuentes de legitimidad: conocimiento, premio, etc.

\section{Conclusión}

El liderazgo en la educación universitaria responde en su caracterización a la pluralidad de modelos complejos que existen en las organizaciones universitarias, tendiendo a ser predominantes dos modelos: el meritocrático o de poder experto y el carismático.

Quedaría pendiente determinar cuál de estos modelos redunda en el ejercicio de un liderazgo efectivo en el marco de la naturaleza peculiar y compleja de anarquía organizada.

\section{Referencias Bibliográficas}

Arias, I, (2012) Revisión histórico-jurídica del principio de Autonomía Universitaria y su relación con la existencia de elecciones en el seno de la Universidad, Dereito Vol.21, N| 1, p.p. 99-149. Recuperado en: www.usc.es/revistas/index.php/dereito/article/download/389/386

Cardona, P. (2000) Liderazgo Relacional, Documento de investigación No 412, Universidad de Navarra, recuperado de: https://www.iese.edu/research/pdfs/DI-0412.pdf

Labourdette, S., \& Scaricabarozzi, R. (2010). Hacia un nuevo concepto de liderazgo. Orientación y sociedad, $\quad \mathrm{N}^{\mathrm{0}} 10, \quad 00 . \quad$ Recuperado de http://www.scielo.org.ar/scielo.php?script=sci_arttext\&pid=S1851$88932010000100001 \& \operatorname{lng}=\mathrm{es} \&$ tlng=es

Marín, A. (Coordinador) (1997) Sociología para la Empresa. McGrawHill editores, Madrid.

Navarra, I., (2 de febrero de 2018), Así se elige un rector. El Espectador. Recuperado de https://www.elespectador.com/opinion/asi-se-elige-un-rector-columna-736961 
Ordorika, I. (1995), Organización, gobierno y liderazgo universitario, en Universidades núm. XLV, pp. 39-48. Recuperado de: https://scholar.google.es/scholar?cluster=11704490982906773123\&hl=es\&as_sdt=2005\&sciodt= 0,5

Ordorika, I. (2001), Aproximaciones teóricas para un análisis del conflicto y el poder en la educación superior, Perfiles educativos vol.23 no.91 p. 76-96. Recuperado de: http://www.scielo.org.mx/scielo.php?script=sci_arttext\&pid=S0185$26982001000100005 \& \operatorname{lng}=\mathrm{es} \& \mathrm{nrm}=\mathrm{iso}$

Shultz, D.P. (1995 Psicología Industrial, McGrawHill editores, Bogotá, Colombia.

Silva, A. (2016) What is Leadership?, Jorunal of Bussiness Studies Quarterly, Vol 8, No 1, p.p. 15, recuperado de: http://docplayer.net/46102564-What-is-leadership-alberto-silva-keiseruniversity.html 